\title{
Correction to: Revision and complication rates in adult shunt surgery: a single institution study
}

\author{
Nadia Mansoor ${ }^{1,2} \cdot$ Ole Solheim $^{1,3} \cdot$ Oddrun A. Fredriksli ${ }^{1,3} \cdot$ Sasha Gulati $^{1,3}$ \\ Published online: 19 January 2021 \\ (C) Springer-Verlag GmbH Austria, part of Springer Nature 2021
}

Acta Neurochirurgica (2020), Oct 31. Online ahead of print https://doi.org/10.1007/s00701-020-04526-z

In the original table 1 there was an error in regard to odds ratio and the $95 \%$ confidence interval for the ICH group (nonrevision group versus revision group).
The odds ratio was given as 0.97 and $95 \%$ CI: $0.9,1.0$. The correct odds ratio and $95 \% \mathrm{CI}$ is 0.3 and $0.0-5.1$, respectively. The correct table 1 is shown below:

Table 1 Patient demographics

\begin{tabular}{|c|c|c|c|c|c|c|c|c|c|}
\hline & \multicolumn{2}{|l|}{ All patients } & \multicolumn{2}{|c|}{ Non-revision group } & \multicolumn{2}{|c|}{ Revision group } & \multirow[t]{2}{*}{$\mathrm{p}$-value } & \multirow[t]{2}{*}{ OR } & \multirow[t]{2}{*}{$95 \% \mathrm{CI}$} \\
\hline & $\mathrm{n}=227$ & $\%$ & $\mathrm{n}=180$ & $\%$ & $\mathrm{n}=47$ & $\%$ & & & \\
\hline Age: mean, yrs $( \pm$ std $)$ & $58.4(15.6)$ & & $59.8(14.9)$ & & $53.0(16.9)$ & & $0.014^{1}$ & & $1.4,12.3$ \\
\hline Gender: Male & 104 & 45.8 & 87 & 48.3 & 17 & 36.1 & 0.136 & 1.7 & $0.9,3.2$ \\
\hline Status (end of follow-up): Dead & 71 & 31.3 & 62 & 34.4 & 9 & 19.1 & 0.044 & 0.45 & $0.2,1.0$ \\
\hline Smoker: Yes & $71^{2}$ & 37.0 & $53^{3}$ & 35.6 & $18^{4}$ & 41.9 & 0.452 & 1.3 & $0.7,2.6$ \\
\hline \multicolumn{10}{|l|}{ Comorbidities } \\
\hline Hypertension & 74 & 32.6 & 63 & 35.0 & 11 & 23.4 & 0.131 & 0.57 & $0.3,1.2$ \\
\hline Diabetes & 23 & 10.1 & 20 & 11.1 & 3 & 6.4 & 0.339 & 0.55 & $0.2,1.9$ \\
\hline COPD & 26 & 11.5 & 22 & 12.2 & 4 & 8.5 & 0.477 & 0.67 & $0.2,2.0$ \\
\hline Cerebrovascular disease & 22 & 9.7 & 16 & 8.9 & 6 & 12.8 & 0.424 & 1.5 & $0.6,4.1$ \\
\hline Cardiovascular disease & 29 & 12.8 & 25 & 13.9 & 4 & 8.5 & 0.325 & 0.6 & $0.2,1.7$ \\
\hline Tumour or malignancy & 34 & 15.0 & 29 & 16.1 & 5 & 10.6 & 0.349 & 0.62 & $0.2,1.7$ \\
\hline $\mathrm{ASA}>2$ & $73^{5}$ & 50.3 & $65^{6}$ & 55.1 & $8^{7}$ & 29.6 & 0.017 & 0.3 & $0.1,0.9$ \\
\hline \multicolumn{10}{|l|}{ Aetiology/Indication } \\
\hline SAH & 81 & 35.7 & 64 & 35.6 & 17 & 36.2 & 0.938 & 1.0 & $0.5,2.0$ \\
\hline NPH & 55 & 24.2 & 50 & 27.8 & 5 & 10.6 & 0.015 & 0.3 & $0.1,0.8$ \\
\hline Tumor or malignancy & 43 & 18.9 & 36 & 20.0 & 7 & 14.9 & 0.426 & 0.7 & $0.3,1.7$ \\
\hline Unspecified HC & 11 & 4.8 & 6 & 3.3 & 5 & 10.6 & 0.038 & 3.5 & $1.0,11.9$ \\
\hline $\mathrm{ICH}$ & 7 & 3.1 & 7 & 3.9 & 0 & 0 & 0.170 & 0.3 & $0.0,5.1$ \\
\hline Traumatic & 8 & 3.5 & 6 & 3.3 & 2 & 4.3 & 0.760 & 1.3 & $0.6,6.6$ \\
\hline Meningitis & 3 & 1.3 & 2 & 1.1 & 1 & 2.1 & 0.587 & 1.9 & $0.2,21.8$ \\
\hline Chiari I & 7 & 3.1 & 3 & 1.7 & 4 & 8.5 & 0.016 & 5.5 & $1.2,25.4$ \\
\hline Other & 6 & 2.6 & 3 & 1.7 & 3 & 6.4 & 0.073 & 3.0 & $0.7,13.9$ \\
\hline Pseudotumor cerebri & 6 & 2.6 & 3 & 1.7 & 3 & 6.4 & 0.073 & 4.0 & $0.8,20.6$ \\
\hline
\end{tabular}

${ }^{1}$ Mann-Whitney $U=3249, p=0.014 .{ }^{2} n=192 .{ }^{3} n=149 .{ }^{4} n=43 .{ }^{5} n=145 .{ }^{6} n=118 .{ }^{7} n=27$.

OD: odds ratio, CI: confidence interval, COPD: chronic obstructive pulmonary disease, SAH: subarachnoid haemorrhage, NPH; normal pressure hydrocephalus, HC; hydrocephalus, ICH: intracerebral haemorrhage. Age both groups: median 61. min 18, max 89, range 71.

The online version of the original article can be found at https://doi.org/10. $1007 / \mathrm{s} 00701-020-04526-\mathrm{z}$

Nadia Mansoor

nadia.mauland.mansoor@stolav.no

1 Department of Neurosurgery, St. Olavs University Hospital, Trondheim, Norway
Nevroklinikken, St. Olavs Hospital, 7006 Trondheim, Norway

3 Department of Neuromedicine and Movement Science, Norwegian University of Science and Technology (NTNU), Trondheim, Norway 